(2) Open Access Full Text Article

\title{
A prospective case series evaluating the safety and efficacy of the Klox BioPhotonic System in venous leg ulcers
}

This article was published in the following Dove Press journal:

Chronic Wound Care Management and Research

15 September 2016

Number of times this article has been viewed

\author{
Andreas Nikolis' \\ Doria Grimard ${ }^{2}$ \\ Yves Pesant ${ }^{3}$ \\ Giovanni Scapagnini ${ }^{4}$ \\ Denis Vézina ${ }^{5}$ \\ 'Division of Plastic Surgery, Victoria \\ Park Research Centre, Montreal, \\ ${ }^{2} \mathrm{Q} \& T$ Research Chicoutimi, \\ Chicoutimi, ${ }^{3}$ St-Jerome Medical \\ Research Inc., St-Jerome, Quebec, \\ Canada; ${ }^{4}$ Department of Medicine and \\ Health Sciences, School of Medicine, \\ University of Molise, Campobasso, \\ Italy; ${ }^{5} \mathrm{Klox}$ Technologies, Laval, \\ Quebec, Canada
}

Purpose: To investigate the safety and efficacy of the BioPhotonic System developed by Klox Technologies in a case series of ten patients with venous leg ulcers.

Patients and methods: Ten patients with chronic venous leg ulcers, having failed on at least one previous therapy, were enrolled into this case series.

Results: Nine patients were evaluable for efficacy. A response (defined as decrease in wound surface area) was observed in seven patients (77.8\%). Of these, four patients (44.4\%) achieved wound closure on average 4 months (127.5 days) following the beginning of the treatment. Two patients did not respond to the investigational treatment. Quality of life improved over time throughout the study. Compliance was excellent, with $93.2 \%$ of visits completed as per protocol. Safety was unremarkable, with only four treatment-emergent-related adverse events, for which no specific intervention was required.

Conclusion: The BioPhotonic System was shown to be safe and extremely well tolerated. It also demonstrated potential in terms of wound closure, wound surface area decrease, and wound bed preparation.

Keywords: biophotonics, light, photobiomodulation, venous leg ulcers

\section{Introduction}

Venous leg ulcers (VLUs) affect a significant proportion of the elderly population, with prevalence rates reported between $3 \%$ and $5 \% .^{1-3}$ While several factors might contribute to the development of leg ulcers, the vast majority of these are due to venous insufficiency. ${ }^{4}$ They represent an important cost to the society ${ }^{5,6}$ and cause significant morbidity, while seriously affecting patients' quality of life. ${ }^{3}$

Despite clinical practice guidelines and many different treatments recommended as standard of care (SOC) for VLUs, an important unmet medical need remains. Literature reports complete wound closure rates between $10 \%$ and $50 \%$ after up to 6 months of treatment, which clearly illustrates the need for new therapies. Recurrence of VLUs is also an issue; reports on dehiscence show that, frequently, a VLU that had been successfully treated will unfortunately break down. ${ }^{7}$

Compression therapy remains the gold standard treatment of VLUs. ${ }^{8,9}$ Many different types of dressings have been developed and commercialized, offering physicians and other health care professionals many options. Management of VLUs may also include skin grafting, ${ }^{10}$ venous surgery, ${ }^{7}$ hyperbaric oxygen therapy, ${ }^{11}$ negative pressure therapy, ${ }^{12}$ and low-level laser therapy, ${ }^{13}$ among others.
Correspondence: Andreas Nikolis Division of Plastic Surgery, Victoria Park Research Centre, 376 Victoria St., Suite 400, Westmount, Quebec, H3ZIC3, Canada

Tel + I 5 I4 4887722

$\mathrm{Fax}+|5| 44883830$

Email anikolis@vicpark.com 


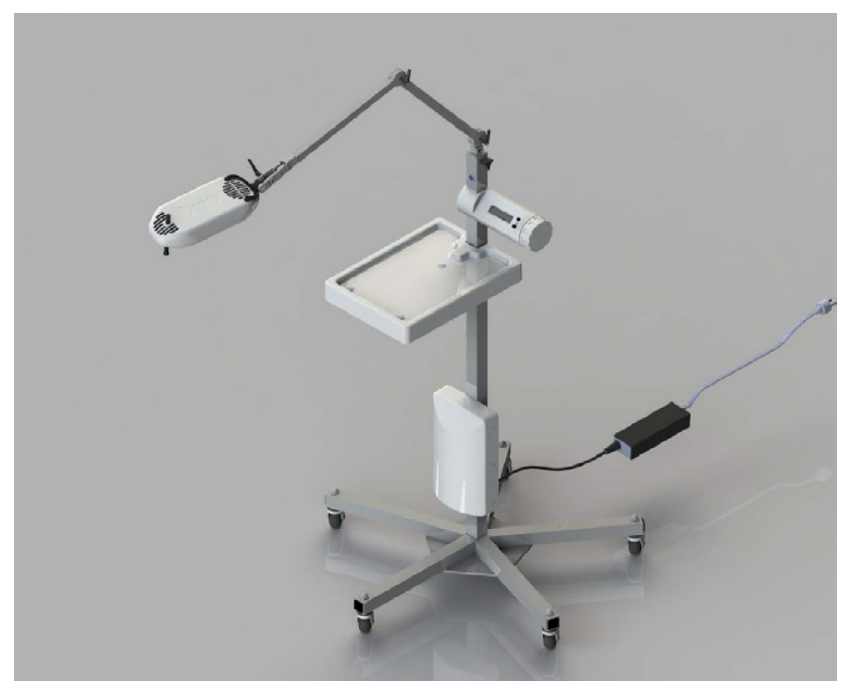

Figure I The Klox multi-LED light.

Abbreviation: LED, light emitting diode.

Klox Technologies has developed a unique medical device composed of a gel and a multi-light emitting diode (LED), which is known to be extremely well tolerated and efficient in the treatment of acne vulgaris. Preclinical data also demonstrated many positive attributes of this system, which was investigated in this case series for the management of VLUs.

\section{Materials and methods Experimental design}

An open-label study design was used to investigate the safety and efficacy of the Klox BioPhotonic System (KBS) in patients with VLUs. Patients were recruited from three different sites. Protocol was approved by Health Canada as well as the Optimum Clinical Research Inc., St-Jerome Hospital Ethics Review Board (NCT02222467). Patients were first screened for eligibility criteria and within 2 weeks started the experimental treatment. The BioPhotonic System was administered twice weekly for 8 weeks and then the investigators could, depending on patients' response and their clinical judgment, either decrease the treatment regimen to once weekly, stay as is (twice weekly), increase to three times per week or give a treatment-pause of 1 or 2 weeks. Patients were treated for a maximum period of 16 weeks or until wound closure. Once their wound closed, the patients were seen again three times over an 8-week period to confirm persistence of wound closure. In all cases, the BioPhotonic System was administered in addition to SOC. When administered more than once weekly, a minimum interval of 3 days was required between treatments.

\section{The Klox BioPhotonic System: photobiomodulation (PBM) and the role of low-energy photons in the wound-healing process}

The KBS represents an innovative treatment for wound healing and skin disorders, and consists of two medical devices: the LED lamp (primary device, Figure 1) and the photo-converter wound gel (secondary device). The topical gel contains specific chromophores, which are not absorbed by the skin, but when excited with the LED lamp, release an ultra-fast micropulsed emission of photons in the form of fluorescence, whose energy delivers wavelengths in the spectra of visible light, from 500 to $610 \mathrm{~nm}$. Together with the activating light (410 to $470 \mathrm{~nm}$ ), these low-energy photons exhibit clinically proven beneficial effect on promoting wound healing.

There has been an increasing amount of biomedical research to substantiate physiological responses to visible light. The first consideration involves the assumption that, for low power visible light to have an effect on a living biological system, the photons must be absorbed by electronic absorption bands belonging to some molecular chromophore or photoacceptor. ${ }^{14}$ The second important consideration involves the use of the definition of PBM as the most suitable term to describe the molecular process and resulting beneficial photobiological responses involved in the treatments of nonthermal low-dose light therapies. ${ }^{15}$ Moreover, even though the therapeutic doses are poorly defined, it should be noted that low-dose light therapies follow a biphasic response that is described by the Arndt-Schulz law, where a weak stimulus can improve a specific biological function and a stronger stimulus abolish the activity or may be toxic. ${ }^{16}$

The use of low levels of visible light has been shown to positively affect each of the phases of wound healing. In the inflammatory phase, the effect of low level-laser irradiation promotes proliferation and degranulation of mast cells. ${ }^{17}$ In the proliferative phase, low-level laser therapy enhances proliferation of various cell lines, including fibroblasts, keratinocytes, osteoblasts, and chondrocytes, as well as induces matrix synthesis. ${ }^{18}$ In the maturation phase, low-power laser phototherapy not only improves reorganization and remodeling of wounds but also restores functional architecture of repaired tissues. ${ }^{19}$ Additional downstream events promoting wound healing through low-dose light therapies are prevention of apoptosis, increase in blood flow, stimulation of angiogenesis, as well as increase in adenosine triphosphate and cyclic adenosine monophosphate. ${ }^{20}$ Furthermore, skin 
exposure to low-level light treatment may start a series of biochemical reactions that result in the production of nitric oxide, a vasodilator and powerful pain reliever, and an antiinflammatory agent. ${ }^{21}$

Another important factor activated by PBM mechanisms is the transforming growth factor- $\beta$. Transforming growth factor- $\beta$ plays a crucial role in the proliferation, resolution, and remodeling of the wound tissue by promoting keratinocytes, endothelial and fibroblast cell migration. ${ }^{22}$ It has also been shown that PBM events may also regulate vascular endothelial growth factor, enhancing the formation of new vessels and improving the healing of skin. ${ }^{23}$ In summary, light treatments can lead to modulation of transcription factors capable of coordinating a wide range of beneficial responses in wound healing.

Different clinical studies have been conducted to investigate low-energy light treatments in the cure of several skin conditions, including rejuvenation of photoaged skin, acne, skin inflammation, and wound healing. ${ }^{24-27}$ Specifically, a PBM approach has shown promising efficacy in VLU treatment. $^{28-30}$

The photo-converter wound gel is presented in two jars, which have to be mixed together just prior to application. Typically, upon evaluation, any excess fibrin or necrotic tissue was debrided. The wound was first cleansed with normal saline and then a $2 \mathrm{~mm}$ thick layer of the gel was applied on the VLU. The wound was then illuminated with the multiLED light for 5 minutes. Once the treatment was completed, the gel was removed from the wound with gentle saline irrigation. A nonadherent dressing was then applied to prevent any contact between the wound and the external environment. Local SOC was then followed: sharp debridement if excess of fibrin, cleansing with normal saline water, compression bandage systems, management of a moist wound environment with nonadherent dressings, management of wound infection, and nutritional assessment.

\section{Imagery system}

At each study visit, images of the VLUs were taken with the Aranz Silhouette ${ }^{\mathrm{TM}}$ Star System (Aranz Medical, Christchurch, New Zealand). This system allows digital pictures to be taken and offers the calculation of the wound surface area, perimeter, volume, average, and maximum depth.

\section{Participants}

Patients had to fulfill all of the following (inclusion) criteria to be eligible for inclusion into the study: 1) male or female 18 years of age and older; 2) the patients or legal guardian must have signed an informed consent form; 3) female of childbearing potential must have a negative pregnancy test result at baseline, and both male and female patients must be willing to adhere to a medically accepted birth control method during the course of the study; 4) willingness to return for all study visits; 5) proven VLU, clinically defined and confirmed by duplex, refilling time or venous hypertension; 6) open VLU for $>4$ weeks prior to study entry; 7) ulcer surface area between 5 and $100 \mathrm{~cm}^{2}$ inclusive, with a maximum depth of $1 \mathrm{~cm}$; the maximum diameter of the wound could not exceed $10 \mathrm{~cm} ; 8)$ wound surface area has not changed by more than $\pm 30 \%$ between screening and first study visits; 9) adequate blood arterial perfusion (ankle-brachial index between 0.7 and 1.3, inclusive).

Patients who met any one of the following (exclusion) criteria on screening visit were not eligible to participate in the study: 1) VLU present for $>12$ months; 2) the ulcer to be treated was planned for operative debridement; 3 ) the ulcer has significant necrotic tissue (eg, $>20 \%$ of the ulcer surface area); 4) major uncontrolled medical disorder(s) such as serious cardiovascular, renal, liver or pulmonary disease, lupus, palliative care, or sickle cell anemia; 5) severe or significant hypoalbuminemia (albuminemia $<30 \mathrm{~g} / \mathrm{L}$, and/or pre-albumin $<5 \mathrm{mg} / \mathrm{dL}$, or hypoproteinemia [proteinemia $<55 \mathrm{~g} / \mathrm{L}]$ ); 6) patients with moderate-to-severe anemia $(\mathrm{Hb}<90 \mathrm{~g} / \mathrm{L}) ; 7)$ patients currently treated for an active malignant disease; 8) patients with history of malignancy within the wound; 9) patients with history of radiation therapy to the wound region; 10) patients with prior diagnosis of active malignant disease who have been disease-free for less than 1 year; 11) patients with a known osteomyelitis or active cellulitis; 12) patients who are immunosuppressed or on high-dose chronic steroid use; 13) patients on systemic corticosteroids (a completion of corticosteroid course at least 30 days prior to study enrolment is required); 14) patients with active or systemic infection (patients could be eligible for rescreening after the systemic infection has subsided); 15) successful revascularization surgery of the leg with the ulcer to be treated $<8$ weeks prior to screening; 16) patients with severely uncontrolled diabetes mellitus (defined as A1C >12\%); 17) Raynaud disease or other severe peripheral microvascular disease; 18) dermatologic comorbid disease (eg, cutis laxa or collagen vascular disease); 19) active bleeding; 20) pregnancy, or breastfeeding; 21) patients with bleeding diathesis; 22) patients on warfarin or intravenous (IV) heparin; 23) patients having any physical or psychiatric condition that in the investigator's opinion would warrant exclusion from the study or prevent the patients from completing the study (eg, severe morbid 
Table I Description of waivers granted

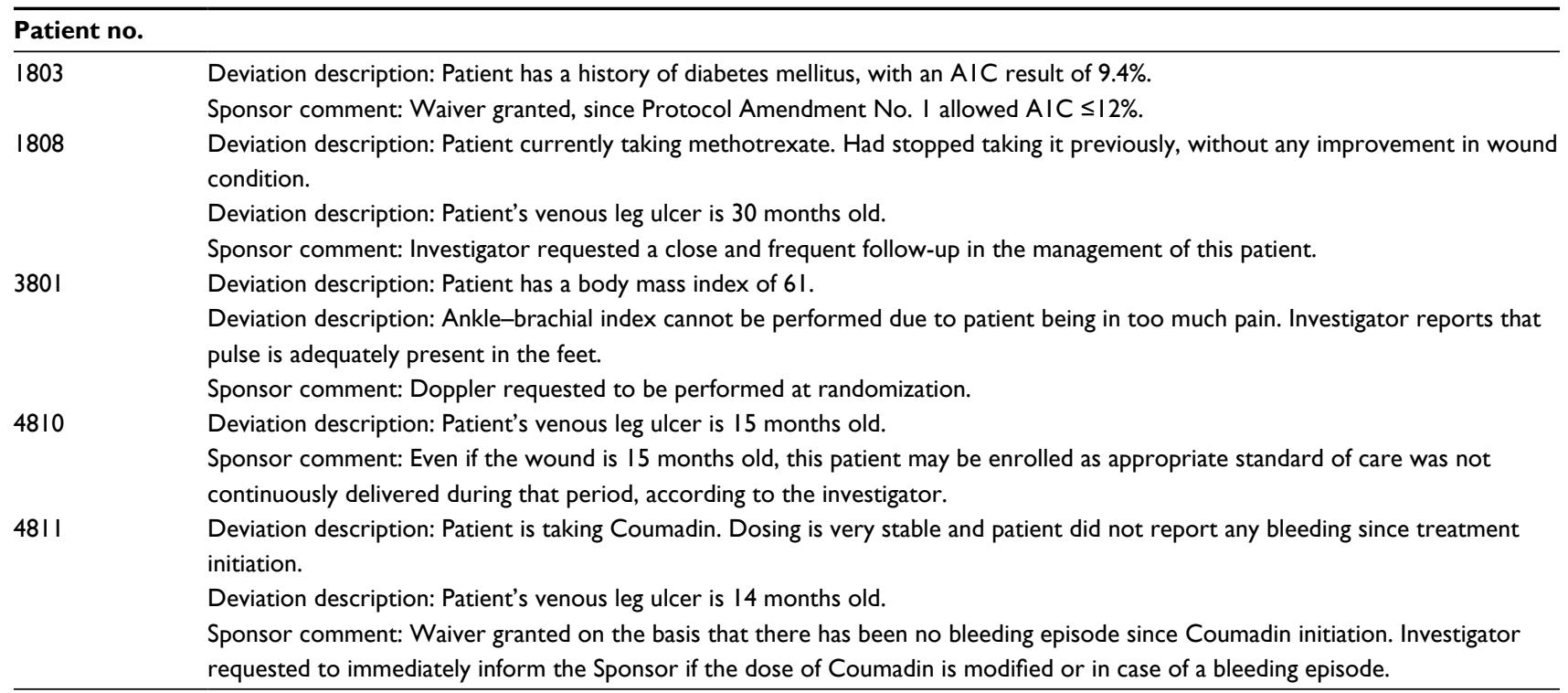

Abbreviation: AIC, glycated hemoglobin.

obesity, recent hip fracture, and suspected noncompliance); 24) patients with ulcers from burns (from exposure to high heat), pressure ulcers, or diabetic foot ulcers; 25) concurrent disease or $\operatorname{drug}(\mathrm{s})$ known to induce severe photosensitivity of the skin, such as porphyria; 26) patients have received biologically based therapy in any wound within 3 months of screening; 27) concurrent participation in another clinical trial involving an investigational drug or device that would interfere with this study; 28) previous participation in another interventional wound healing clinical investigation within the last 60 days prior to screening.

\section{Quality of life}

The Cardiff Wound Impact Schedule ${ }^{\circledR}$ was used to assess the impact of the experimental treatment on patients' quality of life. It was administered at baseline, week 8 , the end of treatment period, as well as the end of the follow-up period.

\section{Efficacy}

Efficacy was assessed through the following parameters: 1) rate of complete wound closure, 2) time to complete wound closure, 3) incidence of wound breakdown, 4) wound surface area reduction over time, 5) wound volume reduction over time, and 6) health-related quality of life.

\section{Safety}

Safety was documented via the collection of 1) adverse events and serious adverse events, 2) device incidents, 3) visual analog scale for pain, 4) clinical laboratory parameters, 5) vital signs, 6) physical examinations, 7) percentage of patients with clinical infection requiring systemic
Table 2 Venous leg ulcers' prior treatments

\begin{tabular}{ll}
\hline Description & $\mathbf{n}(\%)$ \\
\hline Dressings (dry, wet, gels, medicated) & $9(90)$ \\
Compression (bands, socks) & $8(80)$ \\
Systemic antibiotherapy & $3(30)$ \\
Topical antibiotics & $2(20)$ \\
Other topical antibiotics & $2(20)$ \\
Surgery & $0(10)$ \\
Negative pressure therapy & 0 \\
No prior treatment & 0 \\
\hline
\end{tabular}

Note: $\mathrm{N}=10$.

antimicrobial therapy, and 8) concomitant medications and treatments.

\section{Results}

\section{Efficacy}

A total of 21 patients were screened for this study. Five of them met all eligibility criteria; for five others, waivers were requested by the investigators. Waivers concerned eligibility criteria only present in the initial nonamended version of the clinical protocol, and with the understanding there were no other treatment modalities available to the treating physician, they were granted by the sponsor. The nature of waivers is presented in Table 1. Five males and five females were enrolled, with an average age of $71.2 \pm 10.9$ (standard deviation, SD) years (range $57-87$ years). They were all Caucasians and were affected by a VLU for $50 \pm 50.7$ (SD) weeks on average (range 9-140 weeks). At screening, the VLUs mean surface area was $9.3 \pm 10.6(\mathrm{SD}) \mathrm{cm}^{2}$ (range $2.5-38.9 \mathrm{~cm}^{2}$ ) and $9.0 \pm 10.8$ (SD) $\mathrm{cm}^{2}$ prior to first treatment (range $2.1-39.2 \mathrm{~cm}^{2}$ ). All patients had failed at least one prior treatment for their VLU; these are described in Table 2 . The most frequently reported 


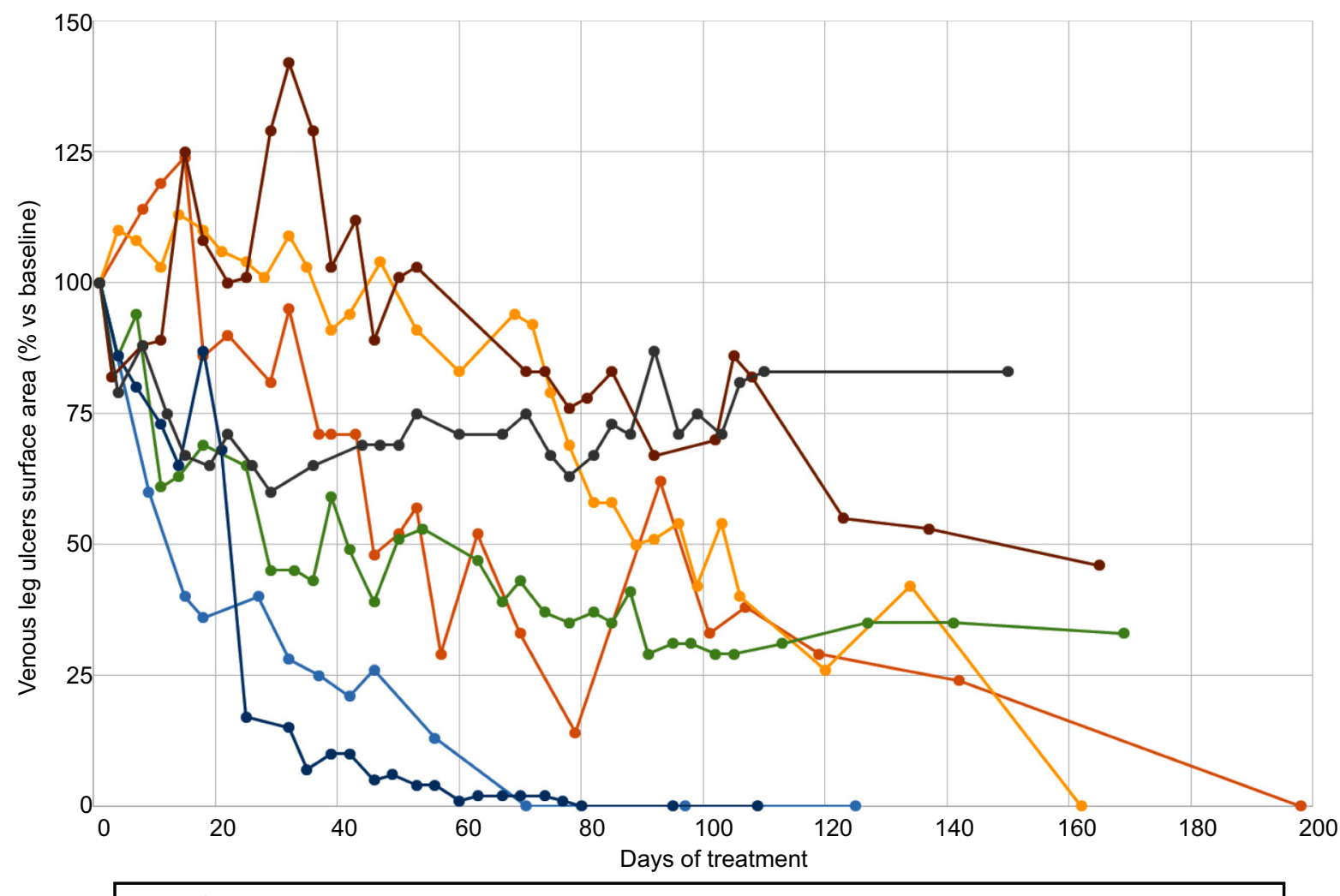

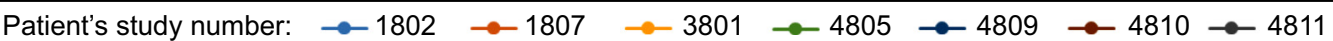

Figure 2 Wounds surface area variation over time (all responders).

comorbidities were bone and joint therapeutic disorders, glucose metabolism disorders, arteriosclerosis, and renal and cardiac disorders.

From the ten enrolled patients, two were discontinued early: one immediately following his first treatment due to pneumonia (unrelated to investigational treatment) and the other following 15 weeks of treatment, due to the need to take a prohibited medication (antibiotic to treat foot cellulitis, also unrelated to investigational treatment).

Nine patients were evaluated for efficacy. A response (defined as a decrease in wound surface area over time) was observed in seven of these nine patients $(77.8 \%$ response rate). From these responders, four (44.4\%) went on to complete wound closure. Time to closure was, on average, 127.5 \pm 61.8 (SD) days (range 71-197 days). The three patients who did not achieve complete wound closure demonstrated a decrease in wound surface area of $46 \%$ (range from $17 \%$ to $67 \%$ ). Figure 2 presents wound surface area reduction over time for each patient. Figures 3-6 present pictures of the four wounds at baseline and end of follow-up period, once closed.

We were able to follow-up two patients who closed their VLU during the course of the study. None of them reported any wound breakdown, with a longest observation period of 53 days. Patients achieving complete wound closure progressed to a $64.5 \%$ surface area reduction within the first 60 days of the study (Table 3). Volume was not accurately represented in the study for two reasons: the superficial nature of VLUs and the lack of sensitivity of the Silhouette ${ }^{\mathrm{TM}}$ Star system for such measurements.

Quality of life was measured in four patients, because of the inclusion of this questionnaire through an amendment at mid-study. Table 4 reports scores obtained by patients over time. All aspects of quality of life (social life, well-being, and physical symptoms and daily living) improved over time, with the most important improvements for the well-being component.

An a posteriori analysis was also conducted at the end of the study, where the evaluation of wound bed preparation was assessed via review of wound pictures. A wound care specialist evaluated the pictures of patients' wound taken at different time points and scored them for granulation tissue and fibrin quantitative presence. Perilesional skin was also examined and assessed for maceration, edema, inflammation, dryness, and overall quality. Based on these parameters, each wound was categorized as potential candidate or not for surgery. Furthermore, when evaluating options for wound closure, five out of eight patients (62.5\%) improved significantly during the treatment period to the point that a skin graft would be tolerated. 


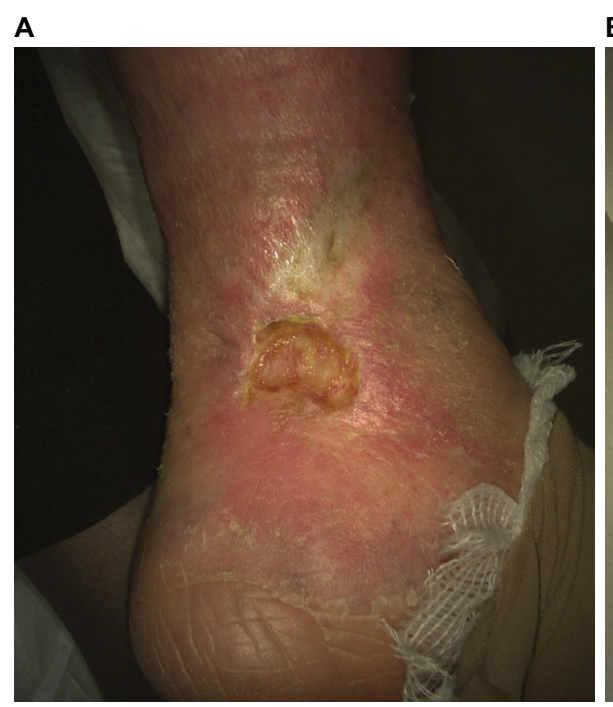

B

Figure 3 Venous leg ulcer of patient no. 1802 at baseline (A) and at last observation (B).

A
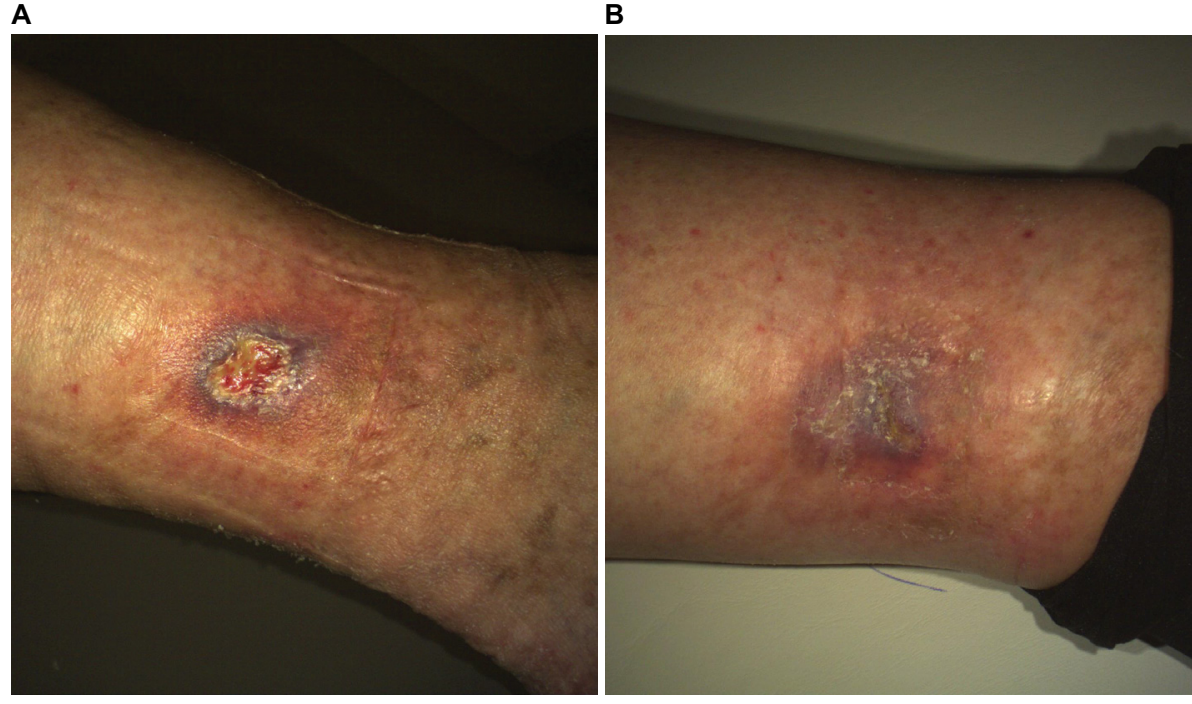

Figure 4 Venous leg ulcer of patient no. 1807 at baseline (A) and at last observation (B).

A
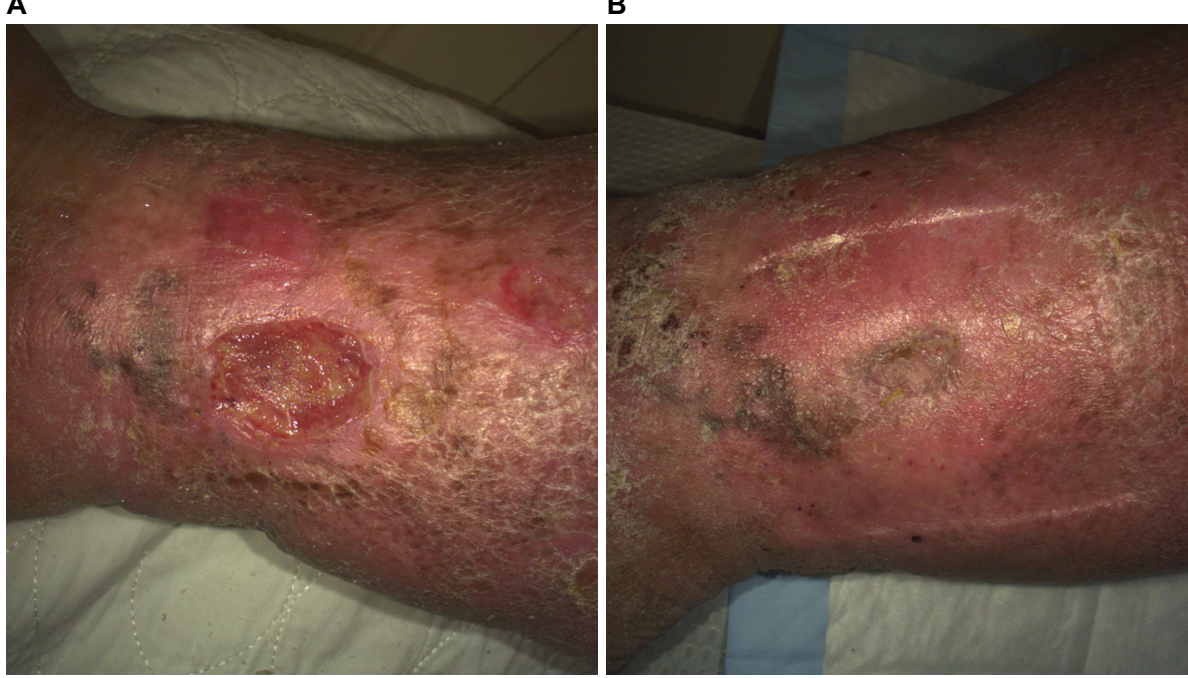

Figure 5 Venous leg ulcer of patient no. $380 \mathrm{I}$ at baseline (A) and at last observation (B). 

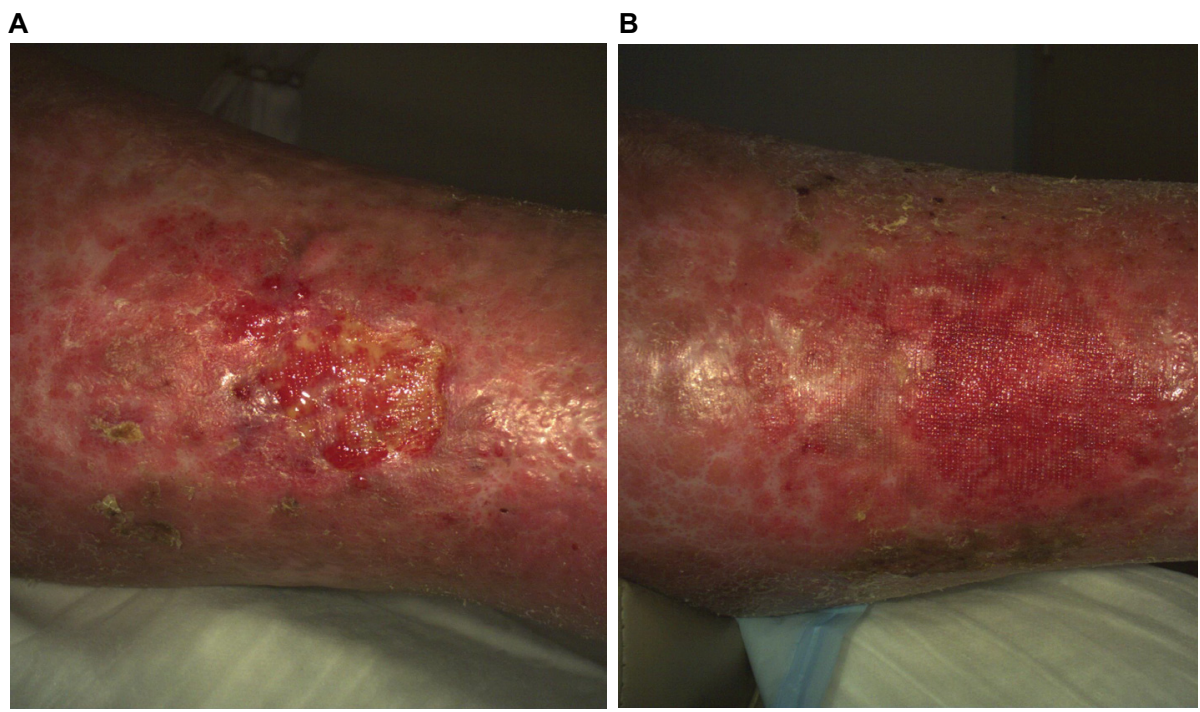

Figure 6 Venous leg ulcer of patient no. 4809 at baseline (A) and at last observation (B)

Table 3 Percentages of wound closure at different time points throughout the study

\begin{tabular}{llllllll}
\hline & \multicolumn{1}{l}{ Day, \% (N) } & & & & \\
\cline { 2 - 8 } & $\mathbf{0}$ & $\mathbf{3 0}$ & $\mathbf{6 0}$ & $\mathbf{9 0}$ & $\mathbf{1 2 0}$ & $\mathbf{1 5 0}$ & $\mathbf{1 8 0}$ \\
\hline All responders & $0.0(7)$ & $32.4(7)$ & $50.1(7)$ & $59.1(7)$ & $61.7(6)$ & $59.0(5)$ & $93.0(1)$ \\
Full responders & $0.0(4)$ & $41.0(4)$ & $66.5(4)$ & $74.2(4)$ & $82.3(3)$ & $80.5(2)$ & $93.0(1)$ \\
\hline
\end{tabular}

Note: Responders defined as patients having shown wound surface area decrease at study end; Full responders defined as patients having closed their wound. ( $\mathrm{N}$ ) represents the number of patients at a given time point.

Table 4 Quality-of-life $\left(\mathrm{CWIS}^{\circledR}\right)$ assessments results

\begin{tabular}{lllll}
\hline Parameters & WI & W8 & WI6 & W24 \\
\cline { 2 - 5 } & Score (N) & Score (N) & Score (N) & Score (N) \\
\hline Social life & $72.0(3)$ & $85.3(4)$ & $79.9(4)$ & $87.5(4)$ \\
Well-being & $29.8(3)$ & $60.7(4)$ & $50.0(4)$ & $64.3(4)$ \\
Physical symptoms & $58.4(3)$ & $89.6(4)$ & $78.4(4)$ & $78.9(4)$ \\
and daily living & & & & \\
\hline
\end{tabular}

Note: The CWIS ${ }^{\circledR}$ questionnaire was introduced through an amendment at midstudy, explaining why not all patients were evaluated. CWIS ${ }^{\circledR}$, Wound Healing Research Unit, University of Wales College of Medicine, Cardiff, UK.

Abbreviations: CWIS ${ }^{\circledR}$, Cardiff Wound Impact Schedule; N, number of patients; W, week.

\section{Safety}

A total of four treatment-emergent-related adverse events were reported (Table 5). One patient reported a heat sensation on face ("feeling hot"), another patient reported pain at the site of application, a third patient reported an increase in burning sensation in the wound area, whereas the fourth one reported skin burning sensation around the ulcer. The pain was reported as severe, whereas the skin burning sensation was reported as moderate; the two other adverse events were of mild intensity. None of them required any specific intervention nor led to study discontinuation. There was no treatment-emergent-related serious adverse event reported throughout the study.

Compliance with study visits was excellent. Overall, $93.2 \%$ of study visits were conducted as per protocol. On
Table 5 Treatment-emergent-related adverse events

\begin{tabular}{|c|c|}
\hline Description & Frequency \\
\hline General disorders and administration site conditions & 2 \\
\hline Feeling hot & I \\
\hline Pain & 1 \\
\hline Injury, poisoning, and procedural complications & 1 \\
\hline Wound complication & I \\
\hline Skin and subcutaneous tissue disorders & 1 \\
\hline Skin burning sensation & I \\
\hline Total & 4 \\
\hline
\end{tabular}

average, investigational treatment duration was 86 days, ranging from 1 to 113 days.

\section{Discussion}

Overall, the KBS demonstrated a promising efficacy profile on the nine patients treated, with seven (out of nine) patients responding favorably to the treatment, for a $77.8 \%$ responder rate. From the ten patients enrolled, one was discontinued early and was not included in the efficacy analyses. From the seven responders, four patients fully closed their ulcers (complete wound closure rate of $44.4 \%$ ) after a mean period of 127.5 days. For the three other responders, based on the trends observed in wound area reduction over time, the patients would have likely continued improving their wound state. Based on the trend observed in wound area reduction over 
time (Figure 2), had treatment been extended, these patients would have likely kept improving their condition over time.

The rate of complete wound closure reported here $(44.4 \%$ within 210 days) compares very well with what is reported in the literature. A PubMed search conducted on October 8, 2015 , to retrieve publications from January 2010 that evaluated VLU treatment identified 27 reports on VLUs response rates period. The closure rates varied from as low as $4 \%$ (at 6 months) up to $90 \%-95 \%$ at 1 year. ${ }^{30-56}$

It is extremely difficult to compare results between studies due to the many variables influencing outcomes, such as patients' age, prior treatments, variability in diagnosis criteria, and wound size. Many factors need to be taken into consideration when conducting such analyses, in order to not over- or underestimate study results.

Patients recruited and treated in our case series had a mean age of 71.2 years, and suffered continuously from a VLU for approximately 1 year (mean of 50.0 weeks). This mean period of 50 weeks is inferior to the 12-month period required in the exclusion criteria 1 , as some of these chronic wounds had a history of closing and reopening during that time. All these wounds had failed at least one previous therapy. Moreover, they were quite large in terms of surface area, with a mean area of $9.0 \mathrm{~cm}^{2}$ (SD of $10.8 \mathrm{~cm}^{2}$, with a maximum size of $39.2 \mathrm{~cm}^{2}$ ) prior to first study treatment application. All these factors, when combined, reinforce the "difficult-to-treat" nature of these wounds.

With a mean time to complete wound closure of 127.5 days, the BioPhotonic System is comparable to similar systems reported in the literature. The same search, as previously described, allowed us to retrieve nine publications reporting on time to wound closure (either mean or median) varying from 5 to 6 weeks up to 1 year. ${ }^{32,36,40,53,57-61}$

The BioPhotonic System performed well when compared with other treatments in terms of closure rates and time to wound closure. Furthermore, its treatment regimen led to a high rate of compliance. In most cases, patients were treated twice weekly, with the possibility of reducing to once weekly. This treatment characteristic has a contributory role in patient compliance, which was excellent in this case series. Overall, $86.2 \%$ of study visits were conducted as planned.

Wound area reduction over time was also assessed in the current case series. When evaluating all responders, at 1 month, wound size had decreased, on average, by $33 \%$, and by $50 \%$ at 2 months. In patients who had wound closure, the wound surface area decreased by two-thirds at 2 months. Again, this compares favorably with the literature, which reports wound size decreases varying from 5\% at 6 weeks to up to $80 \%-90 \%$ at months $3-4 .^{30,45,51,54,55,58,62-69}$
The Silhouette ${ }^{\mathrm{TM}}$ system, although excellent at measuring surface area, was limited in measuring wound volume in our VLU patients.

Once closed, it is of critical importance for the ulcer to remain closed. Wound breakdown, or dehiscence, is a frequent complication observed in the management of venous ulcers. Increased breakdown leads to increased wound infection and impacts the quality of life as the patient returns to baseline in their wound management. In our study, we were able to assess the perseverance of wound closure in two patients, since they completed the follow-up period designed to measure this effect. No case of wound breakdown was observed and anecdotal reports also confirmed the absence of dehiscence in the two other patients who had ulcer closure during the follow-up period. This is again of particular interest; the maintenance of closure potentially offered with the BioPhotonic System might very well be another key benefit for both the patient and the health care professionals.

Health-related quality of life was assessed using the Cardiff Wound Impact Schedule ${ }^{\circledR}$, a questionnaire specifically designed for patients affected by chronic wounds. This questionnaire was chosen for its consistency, ability to discriminate between health states, and good reproducibility. ${ }^{70}$ Self-administered, this validated questionnaire investigates three main components of patients' quality of life: social life, well-being, and physical symptoms and daily living. As reported in Table 4, not only was there no negative impact caused by the KBS on any of these components but there was, in fact, an improvement in each of these components over time.

Debridement was captured throughout the case series. As reported in the literature, this is an important step in wound healing management. ${ }^{71}$ The removal of excess fibrin and necrotic skin, which inhibit the development of healthy new tissue, is of critical importance, as susceptibility to infection increases. Furthermore, necrotic tissues can also mask underlying infections.

Sharp debridement was allowed in our clinical study, prior to study treatments. Overall, debridement was performed in $31.8 \%$ of visits. All patients except one underwent debridement, with a frequency varying from $7 \%$ to $90 \%$ of the visits among patients requiring repeated debridements. Superficial or deep debridement did not interfere with the Klox BioPhotonic System in the management of VLUs.

In addition to the impact on wound closure, is the potential role of biophotonics in wound bed preparation. Part of TIME (tissue, infection, moisture, and wound edge), wound bed preparation ${ }^{72}$ is a concept aimed at optimizing conditions 
at the wound bed so as to encourage normal endogenous healing. The BioPhotonic System, through a retrospective review of pictures, was shown to have permitted up to $62.5 \%$ of wounds to potentially be treated with skin grafting at one point during the course of the study, independently of wound size. It is important to note that all wounds included in this study had failed previous therapies. The concept of managing difficult-to-treat wounds with wound bed preparation protocol in combination with surgery should be evaluated.

This same system (LumiHeal ${ }^{\mathrm{TM}}$, Klox Technologies Inc, Laval, Quebec, Canada) has recently been introduced on the Italian market, following the CE Mark granted by the European Community. Real-life experience gathered so far is similar to what was observed in the present study, that is, a very good safety profile with great efficacy at reducing wound area, even up to closure. It is important to note that the intended use in Europe is not only for the treatment of VLUs but also for pressure ulcers, diabetic foot ulcers, and acute wounds, including cosmesis and function.

In this case series, the use of the BioPhotonic System was coupled with a very favorable safety profile. A total of four treatment-emergent-related adverse events were reported, leading to an incidence of one treatment-emergent-related adverse event over $55(0.02 \%)$ treatments across all patients. From these, two were reported to be of mild intensity (feeling hot and skin burning), one was of moderate intensity (skin burning sensation), whereas pain was reported as severe. None of them required any specific intervention and did not lead to study discontinuation. There was no treatmentemergent-related serious adverse event. Furthermore, the investigational treatment did not cause any abnormal values in laboratory analyses (biochemical, hematological, or in urine). There was no clinically significant impact on vital signs and no negative impact on any constituents of the physical examinations.

Of note, during treatment with the BioPhotonic System, there were no cases that developed a clinical infection requiring systemic antimicrobial therapy. This is particularly important when we consider the at-risk nature of an open wound. Fagerdahl et $\mathrm{al}^{73}$ reported a complication rate of $21 \%$ with negative pressure therapy in a retrospective study involving 87 wounds of different etiologies. To date, $\sim 200$ patients have been treated under different study protocols with the BioPhotonic platform, with no treatment-related infection or treatment-related serious adverse event.

Tolerability was also assessed through the use of a visual analog scale for pain, at each study visit, and before and after treatment. There was a gradual decrease in quantifiable pain felt by the patient over time. There was no difference in terms of pain severity reported before vs immediately following treatment.

The safety profile reported in this case series is very similar to what has been observed in two patients with VLUs treated under the Health Canada Special Access Program, where no treatment-related serious adverse events were reported. This same safety profile was also confirmed in other medical conditions, with similar BioPhotonic Systems. When compared with other treatments used in the management of VLUs, the safety of the BioPhotonic System is certainly an added benefit, offering peace of mind to the treating physician.

There are some limitations in this case series that should be noted such as the absence of a control group and the assessment of quality of life. The addition of a control group would have limited the number of patients in each group and made recruitment challenging. It was deemed a priority to evaluate the impact of the technology on VLU in a smaller proof-of-concept study and use necessary control groups in a larger trial evaluating efficacy against placebo and possibly another established modality. Furthermore, strict eligibility criteria were used to minimize any external factors that may have influenced the wound healing process.

\section{Conclusion}

The use of the BioPhotonic System in the treatment of VLUs was shown to be extremely safe and very well tolerated by our patient population. The system was efficacious, with a responder rate of $77.8 \%$ and a closure rate of $44.4 \%$. It also allowed for the progression of wounds sufficiently for patients to undergo surgery in $62.5 \%$ of cases. Easy to administer, this medical device has all the required characteristics to become a treatment of choice in the management of chronic VLUs.

\section{Acknowledgments}

The authors want to thank the investigational site study personnel, all the colleagues who collaborated in this project, and all the study patients for their participation.

\section{Disclosures}

AN is consultant with Klox Technologies. DV was an employee of Klox Technologies. The other authors do not report any conflicts of interest in this work.

\section{References}

1. Margolis DJ, Bilker W, Santanna J, Baumgarten M. Venous leg ulcer: incidence and prevalence in the elderly. J Am Acad Dermatol. 2002;46(3):381-386. 
2. Canadian Institute for Health Information. Compromise Wounds in Canada; 2013

3. Hopman WM, Buchanan M, VanDenKerkhaf EG, Harrison MB. Pain and health-related quality of life in people with chronic leg ulcers. Chronic Dis Inj Can. 2013;33(3):167-174.

4. Collins L, Seraj S. Diagnosis and treatment of venous ulcers. Am Fam Physician. 2010;81(8):989-996.

5. Ruckley CV. Socioeconomic impact of chronic venous insufficiency and leg ulcers. Angiology. 1997;48(1):67-69.

6. Ragnarson Tennvall G, Hjelmgren J. Annual costs of treatment for venous leg ulcers in Sweden and the United Kingdom. Wound Repair Regen. 2005;13(1):13-18.

7. Barwell JR, Davies CE, Deacon J, et al. Comparison of surgery and compression with compression alone in chronic venous ulceration (ESCHAR study): randomized controlled trial. Lancet. 2004;363(9424):1854-1859.

8. O'Donnell TF, Passman MA, Marston WA, et al. Management of venous leg ulcers: Clinical practice guidelines of the society for vascular surgery ${ }^{\circledR}$ and the American venous forum. J Vasc Surg. 2014;60:3S-59S.

9. Harding K, Dowsett C, Fias L, et al. Simplifying venous leg ulcers management. Consensus recommendations; 2015. Available from: $\mathrm{http} / / / \mathrm{www}$.woundsinternational.com/consensus-documents/view/ simplifying-venous-leg-ulcer-management. Accessed July 15, 2016.

10. Jones JE, Nelson EA, Al-Hity A. Skin grafting for venous leg ulcers. Cochrane Database Syst Rev. 2013;30(1):CD001737.

11. Kranke P, Bennett MH, Martyn-St James M, Schnabel A, Debus SE, Weibel S. Hyperbaric oxygen therapy for chronic wounds. Cochrane Database Syst Rev. 2015;6:CD004123.

12. Dumville JC, Land L, Evans D, Peinemann F. Negative pressure wound therapy for treating leg ulcers. Cochrane Database Syst Rev. 2015;7:CD011354.

13. Flemming KA, Cullum NA, Nelson EA. A systematic review of laser therapy for venous leg ulcers. J Wound Care. 1999;8(3):111-114.

14. Sutherland JC. Biological effects of polychromatic light. Photochem Photobiol. 2002;76:164-170.

15. Kim HP. Lightening up light therapy: Activation of retrograde signaling pathway by photobiomodulation. Biomol Ther (Seoul). 2014;22(6):491-496.

16. Huang YY, Sharma SK, Carroll J, Hamblin MR. Biphasic dose response in low level light therapy - an update. Dose Response. 2011;9(4): 602-618.

17. Fathabadie FF, Bayat M, Amini A, Bayat M, Rezaie F. Effects of pulsed infra-red low level-laser irradiation on mast cells number and degranulation in open skin wound healing of healthy and streptozotocin-induced diabetic rats. J Cosmet Laser Ther. 2013;15(6):294-304.

18. AlGhamdi KM, Kumar A, Moussa NA. Low-level laser therapy: a useful technique for enhancing the proliferation of various cultured cells. Lasers Med Sci. 2012;27(1):237-249.

19. Enwemeka CS, Parker JC, Dowdy DS, Harkness EE, Sanford LE, Woodruff LD. The efficacy of low-power lasers in tissue repair and pain control: a meta-analysis study. Photomed Laser Surg. 2004;22(4):323-329.

20. Avci P, Gupta A, Sadasivam M, Vecchio D, Pam Z, Pam N, Hamblin MR. Low-level laser (light) therapy (LLLT) in skin: stimulating, healing, restoring. Semin Cutan Med Surg. 2013;32(1):41-52.

21. Mittermayr R, Osipov A, Piskernik C, et al. Blue laser light increases perfusion of a skin flap via release of nitric oxide from hemoglobin. Mol Med. 2007;13(1-2):22-29.

22. Arany PR, Nayak RS, Hallikerimath S, Limaye AM, Kale AD, Kondaiah P. Activation of latent TGF-beta1 by low-power laser in vitro correlates with increased TGF-beta1 levels in laser-enhanced oral wound healing. Wound Repair Regen. 2007;15:866-874.

23. Cury V, Moretti AI, Assis L, et al. Low level laser therapy increases angiogenesis in a model of ischemic skin flap in rats mediated by VEGF, HIF-1 $\alpha$ and MMP-2. J Photochem Photobiol B. 2013;125:164-170.

24. Weiss RA, McDaniel DH, Geronemus RG, Weiss MA. Clinical trial of a novel non-thermal LED array for reversal of photoaging: clinical, histologic, and surface profilometric results. Lasers Surg Med. 2005;36(2):85-91.
25. Goldberg DJ, Russell BA. Combination blue $(415 \mathrm{~nm})$ and red (633 $\mathrm{nm})$ LED phototherapy in the treatment of mild to severe acne vulgaris. J Cosmet Laser Ther. 2006;8(2):71-75.

26. Lim W, Lee S, Kim I, et al. The anti-inflammatory mechanism of $635 \mathrm{~nm}$ light-emitting-diode irradiation compared with existing COX inhibitors. Lasers Surg Med. 2007;39(7):614-621.

27. Iordanou P, Baltopoulos G, Giannakopoulou M, Bellou P, Ktenas E. Effect of polarized light in the healing process of pressure ulcers. Int $J$ Nurs Pract. 2002;8(1):49-55.

28. Medenica L, Lens M. The use of polarised polychromatic non-coherent light alone as a therapy for venous leg ulceration. $J$ Wound Care. 2003;12(1):37-40.

29. Caetano KS, Frade MA, Minatel DG, Santana LA, Enwemeka CS. Phototherapy improves healing of chronic venous ulcers. Photomed Laser Surg. 2009;27(1):111-118.

30. Landau Z, Migdal M, Lipovsky A, Lubart R. Visible light-induced healing of diabetic or venous foot ulcers: a placebo-controlled double-blind study. Photomed Laser Surg. 2011;29(6):399-404.

31. Brizzio E, Amsler F, Lun B, Blättler W. Comparison of low-strength compression stockings with bandages for the treatment of recalcitrant venous ulcers. J Vasc Surg. 2010;51(2):410-416.

32. Escaleira R, Cardoso M, Rego J, Macedo P, Midões A. Efficacy of a two-component compression system for the therapy of venous leg ulcers. J Wound Care. 2010;19(3):104-109.

33. Leclère FM, Puechguiral IR, Rotteleur G, Thomas P, Mordon SR. A prospective randomized study of $980 \mathrm{~nm}$ diode laser-assisted venous ulcer healing on 34 patients. Wound Repair Regen. 2010;18(6):580-585.

34. Milic DJ, Zivic SS, Bogdanovic DC, et al. The influence of different sub-bandage pressure values on venous leg ulcers healing when treated with compression therapy. J Vasc Surg. 2010;51(3):655-661.

35. Ortega-Zilic N, Hunziker T, Läuchli S, et al. EpiDex ${ }^{\circledR}$ Swiss field trial 2004-2008. Dermatology. 2010;221(4):365-372.

36. Romanelli M, Dini V, Bertone MS. Randomized comparison of OASIS wound matrix versus moist wound dressing in the treatment of difficultto-heal wounds of mixed arterial/venous etiology. Adv Skin Wound Care. 2010;23(1):34-38.

37. Teo TK, Tay KH, Lin SE, et al. Endovenous laser therapy in the treatment of lower-limb venous ulcers. J Vasc Interv Radiol. 2010;21(5): 657-662.

38. Hokkam E, El-Labban G, Shams M, Rifaat S, El-Mezaien M. The use of topical phenytoin for healing of chronic venous ulcerations. Int $J$ Surg. 2011;9(4):335-338.

39. Belcaro G, Cesarone MR, Errichi BM, et al. Venous and diabetic ulcerations: management with topical multivalent silver oxide ointment. Panminerva Med. 2010;52(2 Suppl 1):37-42.

40. Watson JM, Kang'ombe AR, Soares MO, et al; VenUS III Team. Use of weekly, low dose, high frequency ultrasound for hard to heal venous leg ulcers: the VenUS III randomised controlled trial. BMJ. 2011;342:d1092.

41. Giuggioli D, Colaci M, Manfredi A, Mariano M, Ferri C. Platelet gel in the treatment of severe scleroderma skin ulcers. Rheumatol Int. 2012;32(9):2929-2932.

42. Kelechi TJ, Mueller M, Hankin CS, Bronstone A, Samies J, Bonham PA. A randomized, investigator-blinded, controlled pilot study to evaluate the safety and efficacy of a poly- $\mathrm{N}$-acetyl glucosamine-derived membrane material in patients with venous leg ulcers. JAm Acad Dermatol. 2012;66(6):e209-215.

43. Lazareth I, Moffatt C, Dissemond J, et al. Efficacy of two compression systems in the management of VLUs: results of a European RCT. $J$ Wound Care. 2012;21(11):553-554, 556, 558 passim.

44. Maggio G, Armenio A, Ruccia F, Giglietto D, Pascone M, Ribatti D. A new protocol for the treatment of the chronic venous ulcers of the lower limb. Clin Exp Med. 2012;12(1):55-60.

45. Meaume S, Truchetet F, Cambazard F, et al; CHALLENGE Study Group. A randomized, controlled, double-blind prospective trial with a Lipido-Colloid Technology-Nano-OligoSaccharide Factor wound dressing in the local management of venous leg ulcers. Wound Repair Regen. 2012;20(4):500-511. 
46. Weller CD, Evans SM, Staples MP, Aldons P, McNeil JJ. Randomized clinical trial of three-layer tubular bandaging system for venous leg ulcers. Wound Repair Regen. 2012;20(6):822-829.

47. Wong IK, Andriessen A, Charles HE, Thompson D, Lee DT, So WK, Abel M. Randomized controlled trial comparing treatment outcome of two compression bandaging systems and standard care without compression in patients with venous leg ulcers. J Eur Acad Dermatol Venereol. 2012;26(1):102-110.

48. Chaby G, Senet P, Ganry O, et al; Angio-Dermatology Group of the French Society of Dermatology. Prognostic factors associated with healing of venous leg ulcers: a multicentre, prospective, cohort study. Br J Dermatol. 2013;169(5):1106-1113.

49. Dolibog P, Franek A, Taradaj J, et al. A comparative clinical study on five types of compression therapy in patients with venous leg ulcers. Int J Med Sci. 2013;11(1):34-43.

50. Harding K, Sumner M, Cardinal M. A prospective, multicentre, randomised controlled study of human fibroblast-derived dermal substitute (Dermagraft) in patients with venous leg ulcers. Int Wound J. 2013;10(2):132-137.

51. Humbert P, Mikosinki J, Benchikhi H, Allaert FA. Efficacy and safety of a gauze pad containing hyaluronic acid in treatment of leg ulcers of venous or mixed origin: a double-blind, randomised, controlled trial. Int Wound J. 2013;10(2):159-166.

52. Ashby RL, Gabe R, Ali S, et al. Clinical and cost-effectiveness of compression hosiery versus compression bandages in treatment of venous leg ulcers (Venous leg Ulcer Study IV, VenUS IV): a randomised controlled trial. Lancet. 2014;383(9920):871-879.

53. Finlayson KJ, Courtney MD, Gibb MA, O’Brien JA, Parker CN, Edwards $\mathrm{HE}$. The effectiveness of a four-layer compression bandage system in comparison with class 3 compression hosiery on healing and quality of life in patients with venous leg ulcers: a randomised controlled trial. Int Wound J. 2014;11(1):21-27.

54. Forlee M, Rossington A, Searle R. A prospective, open, multicentre study to evaluate a new gelling fibre dressing containing silver in the management of venous leg ulcers. Int Wound J. 2014;11(4):438-445.

55. Harding K, Aldons P, Edwards H, et al. Effectiveness of an acellular synthetic matrix in the treatment of hard-to-heal leg ulcers. Int Wound J. 2014;11(2):129-137.

56. Arenberger P, Elg F, Petyt J, Cutting K. Expected outcomes from topical haemoglobin spray in non-healing and worsening venous leg ulcers. $J$ Wound Care. 2015;24(5):228, 230-232, 236.

57. Vanscheidt W, Harding K, Téot L, Siebert J. Effectiveness and tissue compatibility of a 12-week treatment of chronic venous leg ulcers with an octenidine based antiseptic--a randomized, double-blind controlled study. Int Wound J. 2012;9(3):316-323.

58. Olyaie M, Rad FS, Elahifar MA, GarkazA, Mahsa G. High-frequency and noncontact low-frequency ultrasound therapy for venous leg ulcer treatment: a randomized, controlled study. Ostomy Wound Manage. 2013;59(8):14-20.
59. Adderley U, Stubbs N. Stockings or bandages for leg-ulcer compression? Nurs Times. 2014;110(15):19-20.

60. Beheshti A, Shafigh Y, Parsa H, Zangivand AA. Comparison of highfrequency and MIST ultrasound therapy for the healing of venous leg ulcers. Adv Clin Exp Med. 2014;23(6):969-975.

61. Evangelista MT, Casintahan MF, Villafuerte LL. Simvastatin as a novel therapeutic agent for venous ulcers: a randomized, doubleblind, placebo-controlled trial. Br J Dermatol. 2014;170(5):1151-1157.

62. Schumann H, Calow T, Weckesser S, Müller ML, Hoffmann G. Waterfiltered infrared A for the treatment of chronic venous stasis ulcers of the lower legs at home: a randomized controlled blinded study. $\mathrm{Br} J$ Dermatol. 2011;165(3):541-551.

63. Alsina-Gibert M, Pedregosa-Fauste S. Amniotic membrane transplantation in the treatment of chronic lower limb ulcers. Actas Dermosifiliogr. 2012;103(7):608-613.

64. Burkiewicz CJ, Guadagnin FA, Skare TL, do Nascimento MM, Servin SC, de Souza GD. Vitamin D and skin repair: a prospective, double-blind and placebo controlled study in the healing of leg ulcers. Rev Col Bras Cir. 2012;39(5):401-407.

65. Dereure O, Czubek M, Combemale P. Efficacy and safety of hyaluronic acid in treatment of leg ulcers: a double-blind RCT. $J$ Wound Care. 2012;21(3):131-132, 134-136, 138-139.

66. Harding K, Gottrup F, Jawień A, et al. A prospective, multi-centre, randomised, open label, parallel, comparative study to evaluate effects of AQUACEL $®$ Ag and Urgotul $®$ Silver dressing on healing of chronic venous leg ulcers. Int Wound J. 2012;9(3):285-294.

67. Meaume S, Perez J, Rethore V, et al. Management of chronic wounds with an innovative absorbent wound dressing. Wound Care. 2012;21(7): 315-316, 318, 320-322.

68. Arenbergerova M, Engels P, Gkalpakiotis S, Dubská Z, Arenberger P. Topical hemoglobin promotes wound healing of patients with venous leg ulcers. Hautarzt. 2013;64(3):180-186.

69. Ghatnekar GS, Grek CL, Armstrong DG, Desai SC, Gourdie RG. The effect of a connexin43-based peptide on the healing of chronic venous leg ulcers: a multicenter, randomized trial. J Invest Dermatol. 2015;135(1):289-298.

70. Price PE, Harding KG. The Cardiff Wound Impact Schedule: the development of a condition specific questionnaire to assess health-related quality of life in patients with chronic wounds. Int Wound J. 2004;1(1):10-17.

71. Milne J. Wound-bed preparation: the importance of rapid and effective desloughing to promote healing. Br J Nurs. 2015;24(Suppl. 20): S52-58.

72. EWMA. Position Document: Wound Bed Preparation in Practice. London: MEP Ltd.; 2004.

73. Fagerdahl AM, Bostrom L, Ulfvarson J, Ottosson C. Risk factors for unsuccessful treatment and complications with negative pressure wound therapy. Wounds. 2012;24(6):168-177.
Chronic Wound Care Management and Research

\section{Publish your work in this journal}

Chronic Wound Care Management and Research is an international, peer reviewed, open access, online journal publishing original research, reviews, editorials, and commentaries on the causes and management of chronic wounds and the major issues related to chronic wound management. Topics also include chronic wounds as comorbidities to other

Submit your manuscript here: https://www.dovepress.com/chronic-wound-care-management-and-research-journal

conditions, patient adherence to therapy, and the economic burden of chronic wounds. The manuscript management system is completely online and includes a very quick and fair peer review system, which is all easy to use. Visit http://www.dovepress.com/testimonials.php to read real quotes from published authors. 